\title{
Single Actuator Control Analysis of a Planar 3DOF Hopping Robot
}

\author{
Nicholas Cherouvim and Evangelos Papadopoulos
}

\begin{abstract}
In this paper we explore the mechanism of energy transfer between the single actuated DOF of a one-legged hopping robot and the remaining unactuated DOFs, during stable running. The concept of the energy transfer mechanism is laid out, after which follows an analytical study. Using this study, an initial controller is derived for the control of a simple SLIP model with friction in the leg and hip, using a single actuator at the hip. We show that while this controller is capable of stable motion for the SLIP model, it does not lead to stable locomotion for the full realistic robot model with pitching body, leg inertia and friction in hip and leg. This indicates that the SLIP model often used for controller design may be unsuitable for this purpose. The necessary modifications are then made to the controller to achieve stable locomotion for the full model, again with a single, easy-to-implement actuator located at the hip. Finally, results are shown from applying the controller to the full model for a wide range of parameters leading to stable motions.
\end{abstract}

Index Terms - control, one-legged hopping robot, underactuated.

\section{INTRODUCTION}

$\mathrm{T}$ HEIS The research area of legged robots measures only a few decades of existence. The severe limitations of wheeled vehicles are obvious, when it comes to transversing the anomalous terrain that comprises large surfaces of our planet and others. Legged robots have the potential of being able to handle steep inclines and negotiate obstacles. The fact that legged robots do not come into contact with all the points of the ground they transverse, as in the case of wheeled vehicles, facilitates their motion over rough terrain. This has made legged robots an area of intensive research.

A number of studies have been made on the passive motion of hopping or bounding robots [1], [2], [3] especially based on the SLIP (Spring Loaded Inverted Pendulum) model with a point mass as body and a massless leg [1]. It has been found that using the right initial conditions, the passive system may execute a cyclic motion using no input torque, given that

Support of this work by the Hellenic General Secretariat for Research and Technology (PENED 2003) and the NTUA Senator Committee of Basic Research, Programme "Protagoras", R.C. No. 10, is acknowledged.

N. Cherouvim is with the Mechanical Engineering Department, National Technical University of Athens, Athens, Greece (e-mail: ndcher@mail.ntua.gr).

E. Papadopoulos is with the Mechanical Engineering Department, National Technical University of Athens, Athens, Greece (phone: +30-210-772-1440; fax: +30-210-772-1455; e-mail: egpapado@central.ntua.gr). the massless leg does not require torque to be brought forward during flight [1].

Also, studies have been carried out that have led to control algorithms for passive one-legged systems [4], [5], [6], [7]. In these, either the leg angle at touchdown has been used as a control input or actuators have been added to the model. In some cases, stable control was achieved using only the leg touchdown angle as a control input [5], [7]. However, these models did not incorporate pitching of the body mass, neither, as they were passive, did they include the mechanisms of energy dissipation found in real robots. Other studies included pitching of the body mass, but also did not include losses and further required two actuators for control [4], [6].

Although stable motions can be achieved using the passive model, in the real-world problem of robot locomotion further complexities are added. Firstly, the pitching of the body mass, often overlooked in studies of passive systems and secondly, the inevitable viscous friction present in all the joints, both the revolute (hip) joint and the prismatic (leg) joint. Due to these reasons, it is important to investigate control methods for more realistic robots.

Legged robots have been constructed and controllers designed that lead to stable locomotion [8], [9], [10], [11]. The first three involve controlling one or four legged robots with two actuators to each leg. In [9], the concepts of replenishing energy in the robot and of energy-based control are presented. In [11], the Scout II quadruped is controlled with only one actuator per leg, although the controller gains must be reconfigured depending on the desired speed. In [12], the energy transfer mechanism from forward to vertical via the leg angle has been described, and a leg angle controller to control velocity was employed.

It is evident that there is a need for a controller, requiring the minimum possible number of actuators (i.e. one per leg), that will provide stable robot motion and in addition will not depend either on the robot initial conditions or the specific robot parameters, such as losses, spring stiffness, etc. For this controller to be implemented on a real robot with only one actuator, a mechanism must be devised for transferring energy from the actuated DOF to the unactuated DOFs.

The aim of this paper is to enhance our understanding of the energy transfer mechanism that exists between the single actuated DOF of a one-legged hopping robot and the remaining unactuated DOFs, during stable running. The 
concept of the mechanism is laid out, followed by an analytical study. Based on this study, an initial controller is derived for the control of a simple SLIP model with friction in the hip and leg, using a single actuator at the hip. While the controller is shown to lead to stable motion for the SLIP model, it does not do so for the full realistic robot model with pitching body, leg inertia and friction in hip and leg. This is a strong indication that the SLIP model, often used for controller design, may actually be unsuitable for this. The necessary modifications are then made to the controller to achieve stable locomotion for the full model, again with a single, easy-to-implement actuator located at the hip. Finally, results are shown from applying the controller to the full model for a wide range of parameters leading to stable motions. This paper may also contribute to the control of other multilegged systems, as the one-legged robot can be seen as part of a more complex system. Finally, it is hoped that it may play a part in enhancing the understanding of underactuated systems.

\section{RoBOT DYNAMICS}

In this paper, references are made to two distinct models. The first model developed corresponds to a realistic robot incorporating a pitching body, inertia in the leg, as well as friction both at the hip and the leg. This model is referred to as the full model. Further, the SLIP model, with a point mass as body, no body pitching and a massless leg is described. This model is often used for controller design.

\section{A. Full Model}

The full model of the robot is shown in Fig. 1. This incorporates the real-world characteristics of a pitching body and inertia in the leg, often neglected in other studies. The body of mass $m$ is considered to have inertia $I_{b}$, while the body's center of mass (CM) is located at the hip joint. The robot leg is equipped with a spring of stiffness $k$ and has a rest length of $L$.

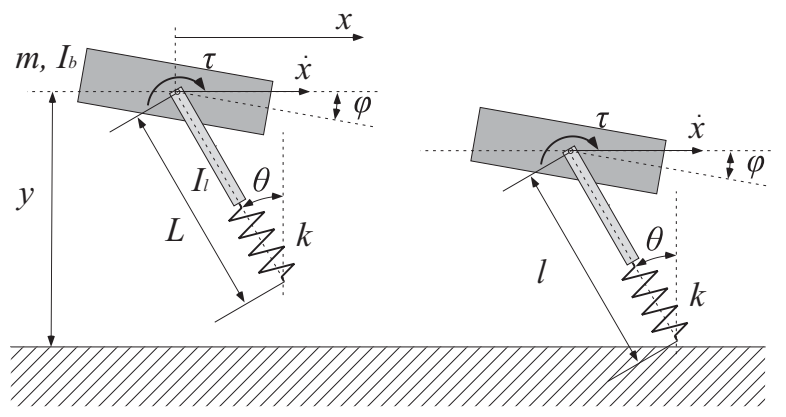

Fig. 1. The robot physical model at the beginning of the motion and at a typical stance configuration.

For the model to be closer to reality, the torque required to bring the leg forward during flight cannot be thought to be zero. However, the mass of a robot leg in comparison to the body mass is typically much smaller. For this reason, the leg is considered to have only inertia $I_{l}$ and no mass. The system also incorporates mechanisms of energy dissipation, due to viscous friction at the leg and at the hip. The viscous coefficients are $b_{l}$ and $b_{h}$ respectively. The robot is equipped with a single actuator capable of exerting torque $\tau$ at the hip joint. The leg forms an angle $\theta$ with the vertical, while the length of the leg at any moment in time is $l$, see Fig. 1. The body forms a pitch angle $\varphi$ with the horizontal. For the configuration in Fig. 1, angle $\theta$ is negative, while angle $\varphi$ is positive.

When moving, the robot goes through a stance and a flight phase, see Fig. 2. During stance, the robot CM covers a distance of $x_{s}$, and during flight a distance of $x_{f}$, reaching an apex height of $h$.

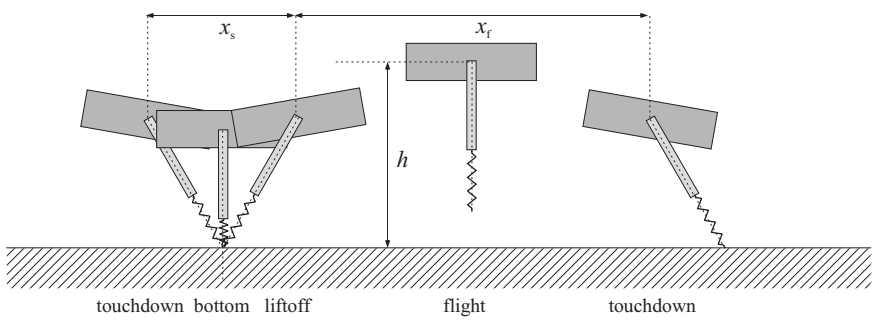

Fig. 2. Phases of the robot motion.

Stance. The robot equations of motion during stance may be found using a Lagrangian approach:

$$
\begin{aligned}
& \left(m l^{2}+I_{l}\right) \ddot{\theta}+2 m l i \dot{\theta}-m g l \sin \theta+b_{h}(\dot{\theta}-\dot{\varphi})=\tau \\
& m \ddot{l}-m l \dot{\theta}^{2}+k(l-L)+m g \cos \theta+b_{l} \dot{l}=0 \\
& I_{b} \ddot{\phi}-b_{h}(\dot{\theta}-\dot{\varphi})=-\tau
\end{aligned}
$$

where $g$ is the acceleration of gravity.

Flight. During flight, the only external force on the robot is gravity. The robot CM position may be determined by the horizontal position $x$ and distance from the ground $y$ (see Fig. 1). The hip actuator may exert a torque to modify the configuration of the system, determined by the angles $\theta, \varphi$. Therefore, the equations of motion during flight are:

$$
\begin{array}{cc}
\ddot{x}=0 & \quad, \quad I_{l} \ddot{\theta}+b_{h}(\dot{\theta}-\dot{\varphi})=\tau \\
\ddot{y}=-g & , \quad I_{b} \ddot{\phi}-b_{h}(\dot{\theta}-\dot{\varphi})=-\tau
\end{array}
$$

\section{B. SLIP Model}

The SLIP model is essentially a simplification of the full model described above. The body is considered to be a point mass, while the leg is massless and has no inertia. Viscous friction is still incorporated in the hip and the leg. The equations of motion for this model are expressed in Eqs. (3), (4), for the stance phase and flight phase respectively. 


$$
\begin{gathered}
m l^{2} \ddot{\theta}+2 m l \ddot{l} \dot{\theta}-m g l \sin \theta+b_{h} \dot{\theta}=\tau \\
m \ddot{l}-m l \dot{\theta}^{2}+k(l-L)+m g \cos \theta+b_{l} \dot{l}=0 \\
\ddot{x}=0 \\
\ddot{y}=-g
\end{gathered}
$$

\section{CONCEPT OF MECHANISM OF ENERGy TRANSFER INTO AXIAL DOF}

Both robot models discussed above are equipped with a hip joint actuator. Having only this actuator is considerably easier to implement than having an actuator exerting axial forces on the leg. However, the system is underactuated and no method of transferring energy directly into the axial direction of the leg exists. Observing the second of Eqs. (1) governing the motion in the axial direction, it can be seen that, due to actuator location, this equation has no input force; hence it must be controlled indirectly. The concept of an energy transfer mechanism for the axial DOF has been laid out in [9], in the case of an actuated axial DOF. Expanding this concept, the transfer mechanism below allows for energy transfer when no axial actuation is available.

It is obvious that compensating for the energy lost due to friction at the hip is simple since the hip joint is actuated. However, compensating for the energy lost in the leg is more complex. A first approach would suggest that the hip joint actuator might increase the total system energy by the amount lost in the leg and hip. But this is not adequate, since it would only lead to a continuous increase of energy in the hip DOF, while the energy in the leg would decrease with each cycle, leading to instability.

Therefore, it is necessary to devise a mechanism of transferring energy from the actuated hip into the axial DOF of the leg. To this end, consider what takes place from an energy point of view at the time the robot leg touches down after the flight phase. At this point, as a result of its previous motion, the robot $\mathrm{CM}$ has a given velocity $v$, see Fig. 3 . The subscript $t d$ denotes the value of a quantity at touchdown.

As seen in Fig. 3, the body velocity at touchdown can be analyzed into two components. The first component is that which corresponds to the rotating motion of the leg about the pivot point at the leg-ground contact, while the second is in the axial direction of the leg. In Fig. 3, it can be seen that the resulting magnitude of each component, for the same robot velocity $v$, is determined by the touchdown angle of the leg. This essentially means that, by regulating the touchdown angle, the distribution of the CM (linear) kinetic energy between the rotating and axial directions may be determined. It may be noted that as the magnitude of $\theta_{t d}$ increases, there is also a small increase of the magnitude of $v$, due to the fact that the leg will touch down a little later. However, this is considered to be negligible.

Using this distribution mechanism, it is possible to choose to transfer an extra amount of kinetic energy into the axial direction, so as to complement the energy lost due to viscous friction. Of course, this leads to a decrease of the energy available to the rotating direction. However, this is easily compensated for, since the hip actuator actuates this DOF. It is evident from the coupling of the DOFs in the equations of motion, that some energy will be transferred between the rotating and axial direction during the stance phase. However, the dominant mechanism used for transferring energy in the controllers presented is the one described above.

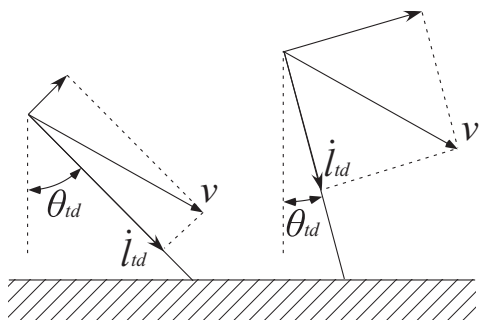

Fig. 3. The robot velocity at touchdown and its distribution for different touchdown angles

To conclude, by using this mechanism, energy can actually be transferred from the actuated hip joint DOF to the unactuated leg axial DOF. All the energy required for complementing system losses will be given by the hip actuator, and then distributed accordingly at touchdown.

\section{MATHEMATICAL FORMULATION OF ENERGY TRANSFERRING MECHANISM}

In this section, the mechanism described above is mathematically formulated to allow its integration into the controller. This is achieved by relating the individual components of the touchdown velocity, $\dot{x}_{t d}$ and $\dot{y}_{t d}$, to the leg angular and linear velocity:

$$
\left[\begin{array}{c}
\dot{\theta}_{t d} \cdot L \\
\dot{l}_{t d}
\end{array}\right]=\left[\begin{array}{cc}
\cos \theta_{t d} & -\sin \theta_{t d} \\
\sin \theta_{t d} & \cos \theta_{t d}
\end{array}\right] \cdot\left[\begin{array}{c}
\dot{x}_{t d} \\
\dot{y}_{t d}
\end{array}\right]
$$

Eq. (5) describes how the velocity is distributed between the rotation $\left(\dot{\theta}_{t d} \cdot L\right.$ component $)$ and the axial direction ( $\dot{l}_{t d}$ component).

Suppose that the robot is executing a steady state motion, composed of a flight phase and a stance phase. Each stance or flight phase is identical to the previous one. Further, let $E_{l}$ be the energy dissipated at the leg during one stance phase. This energy must be replenished in order for the steady state motion to continue. Using the mechanism described above, this means that the leg angle at touchdown must be regulated so that an amount of energy equal to the leg friction losses of the current stance phase is added to the axial leg direction. The slight difference in the robot potential energy at touchdown, for different values of $\theta_{t d}$, is considered to be negligible. Hence, the increase in energy in the leg axial direction must be made in kinetic energy. This may be expressed as: 


$$
\frac{1}{2} m i_{t d, i}^{2}=\frac{1}{2} m i_{l o, i-1}^{2}+E_{l}
$$

where the subscript $l o$ denotes the value of a quantity at liftoff, $i$ denotes the stance phase about to begin, $i-1$ denotes the previous stance phase. From Eq. (6), it follows that, for enough extra energy to be transferred to the leg DOF to cover dissipation losses, the necessary touchdown velocity in the axial direction is:

$$
i_{t d, i}=-\sqrt{i_{l o, i-1}^{2}+\frac{2}{m} E_{l}}
$$

This velocity can be achieved by regulating the touchdown angle as described above. Using Eq. (5), the desired touchdown angle $\theta_{t d, d e s}$ necessary to achieve the desired leg velocity $i_{t d, i}$ can be found. To provide a simple expression for the desired touchdown angle, $\theta_{t d, d e s}$ is presumed to be small enough so that:

$$
\begin{aligned}
& \sin \theta_{t d, d e s} \simeq \theta_{t d, d e s} \\
& \cos \theta_{t d, d e s} \simeq 1-\frac{1}{2} \theta_{t d, d e s}^{2}
\end{aligned}
$$
be:

Now, combining Eqs. (5) and (8), $\theta_{t d, d e s}$ can be found to

$$
\theta_{t d, d e s}=-\frac{\dot{x}_{t d}-\sqrt{\dot{x}_{t d}^{2}+2 \dot{y}_{t d}\left(-\dot{l}_{t d, i}+\dot{y}_{t d}\right)}}{\dot{y}_{t d}}
$$

Using the above touchdown angle, the leg touchdown axial velocity is adequately increased in comparison to the leg velocity at liftoff of the previous stance phase. The difference in velocities leads to a sufficient increase in the energy in the direction of the leg to complement the dissipated energy in the leg $E_{l}$.

In Eq. (9), the expression for the desired touchdown angle is a function of the desired axial velocity at touchdown $\dot{i}_{t d}$. From Eq. (7), $i_{t d}$ is seen to depend on the energy dissipated in the leg $E_{l}$. Since this cannot be measured easily, an analytical approximation is used. In [13] an analytical approximation has been found for the energy dissipated during the stance phase in a leg with viscous friction:

$$
E_{l}=b_{l} p
$$

where $p$ was found to be:

$$
\begin{aligned}
p & =\frac{1}{4}\left(\frac{m}{k}\right)^{3 / 2}\left[2 \sqrt{\frac{k}{m}} g i_{l o, i-1}\left(1-\cos \left(2 \sqrt{\frac{k}{m}} T_{s}\right)\right)\right. \\
& +\left(-g^{2}+\frac{k}{m} i_{l o, i-1}^{2}\right) \sin \left(2 \sqrt{\frac{k}{m}} T_{s}\right) \\
& \left.+2 \sqrt{\frac{k}{m}} T_{s}\left(g^{2}+\frac{k}{m} \dot{l}_{l o, i-1}^{2}\right)\right]
\end{aligned}
$$

where $T_{s}$ is the duration of the stance phase and all other parameters have been defined previously.

\section{SLIP MOdEL CONTROLLER}

In this section a controller, for the SLIP robot model described in Section II, will be derived based on the energy transfer mechanism laid out in the previous section. This controller will be referred to as SL-C.

The desired touchdown angle of the robot for each hop is determined by Eq. (9). This ensures that adequate energy is transferred to the unactuated leg. Since the SLIP model has a massless leg, no torque is required to bring the leg forward during flight. If the robot starts its motion in the flight phase (i.e. with a throw), then for the first touchdown angle, Eq. (9) cannot be used. Therefore for the first touchdown only, the desired touchdown angle is chosen according to the neutral point control method established in [8]:

$$
\theta_{t d, d e s}=-\arcsin \left(\frac{T_{s} \dot{x}_{t d}}{2 L}\right)
$$

This angle represents half the angle the leg would cover during stance if the horizontal velocity of the body were constant.

However, even though energy will be transferred to the axial DOF, the total robot energy will diminish unless energy is provided from some external source to the mechanical system. Using the hip actuator, it is possible to provide energy to the rotating direction, thus increasing the total system energy. The energy input by the actuator is then distributed as necessary between the rotating and axial direction, according to the mechanism described above.

To keep the total system energy constant, the actuator applies the necessary torque during the stance phase. After touchdown and until the leg reaches the vertical position, the torque applied is constant and equal to the energy dissipated in the leg during the stance phase, over the touchdown angle of the leg. When the leg has passed the vertical position, the torque has the form of a proportional controller with the system energy as feedback. Such a proportional energy controller has been used in [9] as part of a control scheme that resulted in stable locomotion for a real robot. In each case an extra term is added to the controller that compensates for the viscous friction at the hip. Therefore the actuator torque during stance is:

$$
\tau=\left\{\begin{array}{cc}
E_{l} / \theta_{t d}-b_{h}(\dot{\theta}-\dot{\varphi}) & \theta<0 \\
k_{1}\left(E_{s y s, d}-E_{s y s}\right)-b_{h}(\dot{\theta}-\dot{\varphi}), & \theta>0
\end{array}\right.
$$

where $E_{s y s, d}$ is the desired system energy, $E_{s y s}$ the current system energy, $k_{1}$ the $\mathrm{P}$ controller gain. The desired system energy is equal to the system energy at the beginning of the motion. The expression of system energy at each moment is: 


$$
E_{s y s}=\frac{1}{2} m \dot{x}^{2}+\frac{1}{2} m \dot{y}^{2}+m g y+\frac{1}{2} k(l-L)^{2}
$$

As will be seen in the results presented in Section VII, the SLIP response to this controller is typically a transient period of about four cycles, followed by stable locomotion.

\section{Full Robot CONTROLLER}

If the derived SLIP controller was to be applied to the case of the full robot model, it would be immediately clear that a steady state motion could not be accomplished using the controller as is. Specifically, gradual instability arises in the body pitching motion, while the total gait is not sustainable. An example of this is shown in Section VII. As mentioned earlier, this result is a strong indication that a more complete robot model than the SLIP is required for controller design, due to the basic qualitative differences in the behavior of the SLIP and the full model.

It is evident that modifications are required to the initial controller presented. The aim of the controller developed here is to be able to start the robot with a wide range of initial conditions without changing the controller gains. It is expected, in accordance with the trial on the SLIP model, that there will be a transitional phase of about four cycles before the steady state motion. The full controller for the original robot will now be presented in three components: (a) the stance phase control, (b) the leg touchdown angle command and (c) the flight phase control. This controller will be referred to as FL-C.

\section{A. Stance phase control}

As mentioned, using the controller as is on the original robot leads to gradual instability in the body pitching motion. To bound the pitching motion of the body, an inverse dynamics controller is used for the body to follow a desired pitching trajectory. During what is estimated to be the transient response period, i.e. the first four hops, the desired trajectory has the form of a sinusoidal counter-oscillation to the oscillation of the leg. The form of this trajectory is based on the analysis of [6]. The desired trajectory is

$$
\phi_{d}=-\frac{I_{l}}{I_{b}} \frac{\theta_{t d, d e s} \sin \left(-\pi \frac{2 t-T_{s}}{T_{s}+T_{f}}\right)}{\sin \left(\pi \frac{T_{f}}{T_{s}+T_{f}}\right)}
$$

where $T_{f}$ is the flight duration, computed in Eq. (24), $T_{s}$ the stance duration. The stance duration is considered to be equal to the stance duration of the previous stance phase, while for the first cycle it is roughly approximated as:

$$
T_{s}=\pi \sqrt{\frac{m}{k}}
$$

After the transient period, the desired trajectory becomes a third order polynomial, enforced by inverse dynamics control. The desired final pitch angle $\varphi_{t d, d e s}$ and pitch velocity $\dot{\varphi}_{t d, d e s}$ are set equal to the pitch angle and velocity at liftoff of the last transient stance phase $\varphi_{l o, l t}, \dot{\varphi}_{l o, l t}$. The trajectory is,

$$
\phi_{d}(t)=a_{4}+a_{5} t+a_{6} t^{2}+a_{7} t^{3}
$$

where

$$
\begin{aligned}
& a_{4}=\phi_{t d, d e s} \\
& a_{5}=\dot{\phi}_{t d, d e s} \\
& a_{7}=\frac{-2 \phi_{l o, l t}+\dot{\phi}_{l o, l t} \cdot T_{s}+2 \phi_{t d, d e s}}{T_{s}^{3}} \\
& a_{6}=\frac{\dot{\phi}_{l o, l t}-\dot{\phi}_{t d, d e s}-3 \cdot T_{s}^{2} a_{7}}{2 \cdot T_{s}}
\end{aligned}
$$

Since the first part of the response is expected to be of a purely transient character, it is evident why Eq. (17) cannot be used from the start of the motion. Also, Eq. (15) cannot be continued after the transitional phase, as it was not found to lead to a stable motion.

For both the transient and steady state desired trajectories, the inverse dynamics controller enforcing them in each case is the same:

$$
\begin{aligned}
\tau=-\left(I_{b} \ddot{\phi}_{d}+k_{d}\left(\dot{\phi}_{d}-\dot{\phi}\right)+k_{p}\left(\phi_{d}-\phi\right)\right. \\
\left.-b_{h}(\dot{\theta}-\dot{\varphi})\right)
\end{aligned}
$$

\section{B. Leg touchdown angle command}

The stance phase control presented above controls the body pitching motion, but replaces the control set out in Section V for keeping the system energy steady, Eq. (13). Therefore, an alternative method must be found to retain the system energy level steady.

To this end, a proportional controller term for the system energy is added to the expression for the desired leg touchdown angle. This term becomes active only after the first four transient cycles. The new expression for the desired touchdown angle, based on Eq. (9), becomes:

$$
\begin{aligned}
\theta_{t d, d e s}= & -\frac{\dot{x}_{t d}-\sqrt{\dot{x}_{t d}^{2}+2 \dot{y}_{t d}\left(-\dot{l}_{t d, i}+\dot{y}_{t d}\right)}}{\dot{y}_{t d}} \\
& +k_{2}\left(-E_{s y s}+E_{s y s, d}\right)
\end{aligned}
$$

where $k_{2}$ is the proportional controller gain. The desired system energy is equal to the value of the system energy after the fourth cycle. The expression for the system energy at each moment is now, 


$$
\begin{aligned}
E_{s y s} & =\frac{1}{2} m \dot{x}^{2}+\frac{1}{2} m \dot{y}^{2}+m g y+\frac{1}{2} k(l-L)^{2} \\
& +\frac{1}{2} I_{l} \dot{\theta}^{2}+\frac{1}{2} I_{b} \dot{\varphi}^{2}
\end{aligned}
$$

It has been observed that the controller performs significantly better when the last two terms, representing the kinetic energy of the leg and body due to inertia, are omitted from the energy calculation. For the first cycle, the desired touchdown angle is chosen as in Eq. (12).

The stabilizing effect of the added term in Eq. (20) on the total system energy is explained as follows. When the system energy is greater than desired, the leg is set further forward for touchdown during the flight phase. Therefore, the resulting velocity in the axial direction of the leg at the beginning of stance is increased, according to Section IV. This results in greater losses in the leg due to viscous friction, according to Eqs. (10), (11). This way the system energy is decreased. Similarly, if the system energy is smaller than the desired energy, then the new term results in an increase of the system energy.

\section{Flight phase control}

To complete the controller, the leg must be brought forward to the desired touchdown angle during flight. Since the robot leg has inertia, the common assumption that the leg may be brought forward with zero torque is not valid. To bring the leg to the desired position, an inverse dynamics controller is adopted for the leg to follow a desired trajectory, ending at the desired touchdown angle. The desired trajectory $\theta_{d}$ is a third order polynomial:

$$
\theta_{d}=a_{0}+a_{1} t+a_{2} t^{2}+a_{3} t^{3}
$$

The parameters of the trajectory are chosen so that the leg has reached the desired angle before the time of touchdown. The parameters are:

$$
\begin{aligned}
& a_{0}=\theta_{l o} \\
& a_{1}=\dot{\theta}_{l o} \\
& a_{3}=\frac{-2 \theta_{t d, d e s}+\dot{\theta}_{l o} \cdot 0.9 \cdot T_{f}+2 \theta_{l o}}{\left(0.9 \cdot T_{f}\right)^{3}} \\
& a_{2}=\frac{-\dot{\theta}_{l o}-3 \cdot\left(0.9 \cdot T_{f}\right)^{2} a_{3}}{2 \cdot\left(0.9 \cdot T_{f}\right)}
\end{aligned}
$$

The duration of the flight phase $T_{f}$ is easily approximated as:

$$
T_{f}=\frac{1}{g}\left(\dot{y}_{l o}+\sqrt{\dot{y}_{l o}^{2}-2 g L\left(\cos \theta_{t d, d e s}-\cos \theta_{l o}\right)}\right)
$$

To implement the controller, the applied torque during flight is:

$$
\begin{aligned}
\tau & =I_{l} \ddot{\theta}_{d}+k_{d}\left(\dot{\theta}_{d}-\dot{\theta}\right)+k_{p}\left(\theta_{d}-\theta\right) \\
& +b_{h}(\dot{\theta}-\dot{\varphi})
\end{aligned}
$$

where $k_{p}, k_{d}$ are the controller gains.

For the complete controller to work on a physical robot, it is necessary to have onboard sensing. The robot velocity and configuration must be known for the calculation of $\theta_{t d, d e s}$ and the feedback of the flight and stance control, as well as for the computation of the system energy in Eq. (20).

\section{RESUltS}

In this section, the response of the SLIP model and the full robot model to the derived controllers will be presented.

\section{A. SLIP model}

SL-C is applied to the SLIP model. A typical response is shown in Fig. 4 , for $m=10 \mathrm{~kg}, k=5000 \mathrm{~N} / \mathrm{m}$. The losses are set to $b_{h}=0.5 \mathrm{~N} \cdot \mathrm{s} / \mathrm{m}$ for the hip and $b_{l}=1 \mathrm{~N} \cdot \mathrm{s} / \mathrm{m}$ for the leg. The robot was started with an initial horizontal velocity of $\dot{x}_{0}=2.2$ $\mathrm{m} / \mathrm{s}$ from a height of $\mathrm{h}=0.7 \mathrm{~m}$. The controller gain is $k_{1}=0.2$.

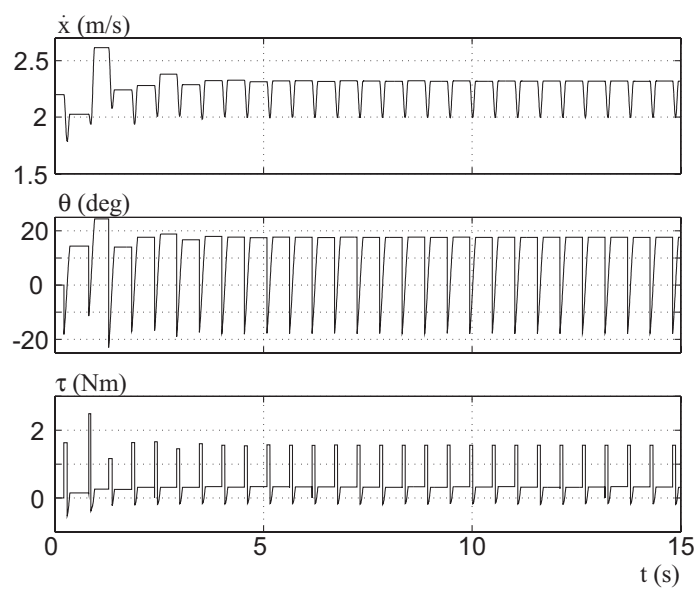

(a)
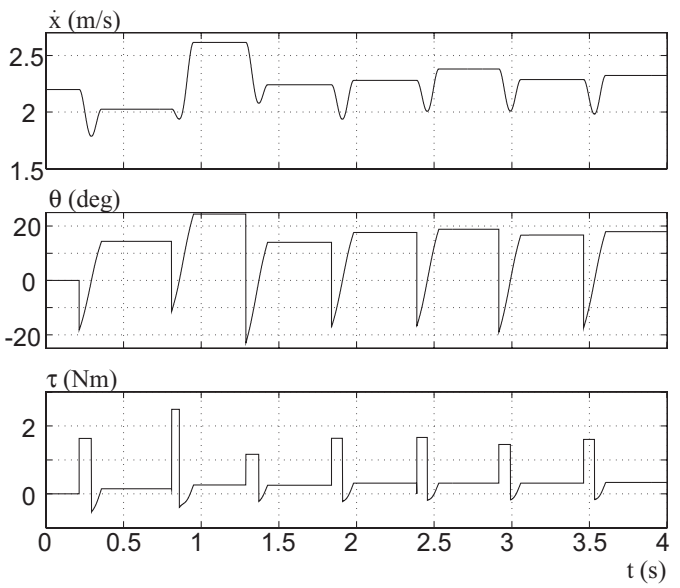

(b)

Fig. 4. Response of the SLIP model to SL-C. (a) The response over $15 \mathrm{~s}$. (b) The transient phase over about $3 \mathrm{~s}$. 
As can be seen in Fig. 4, there is a short transient phase during which the robot states at a characteristic cycle point (i.e. apex) vary considerably. This lasts for the first four or five hops, after which the robot settles into a steady state behavior.

\section{B. Full model}

Here, the response of the full robot is examined. Initially, SL$\mathrm{C}$ is applied to the full model. The resulting response is then compared to the response of the SLIP model under the same controller, shown in Fig. 4. This way, the differences in qualitative behavior can be investigated, between the full model and the SLIP model, often used for controller design.

For the application of SL-C to the full model, the same model parameters and initial conditions are used as in the previous example for the SLIP model (Fig. 4). The only difference in the controller is that a torque is now necessary to bring the leg forward during flight. Also, the remaining full model parameters are $I_{b}=0.5 \mathrm{~kg} \mathrm{~m}^{2}, I_{l}=0.05 \mathrm{~kg} \mathrm{~m}^{2}$. The response is shown in Fig. 5.

As can be seen, although the exact same controller configuration worked perfectly on the SLIP, it is not adequate for the full model. The pitch angle quickly tends to instability, while the leg angle behavior has the form of a gradual decay. It is evident that the motion is not sustainable. This is a display of the fundamental differences between the properties of the SLIP and the realistic full model and shows that a controller designed using the SLIP model fails in a realistic case.
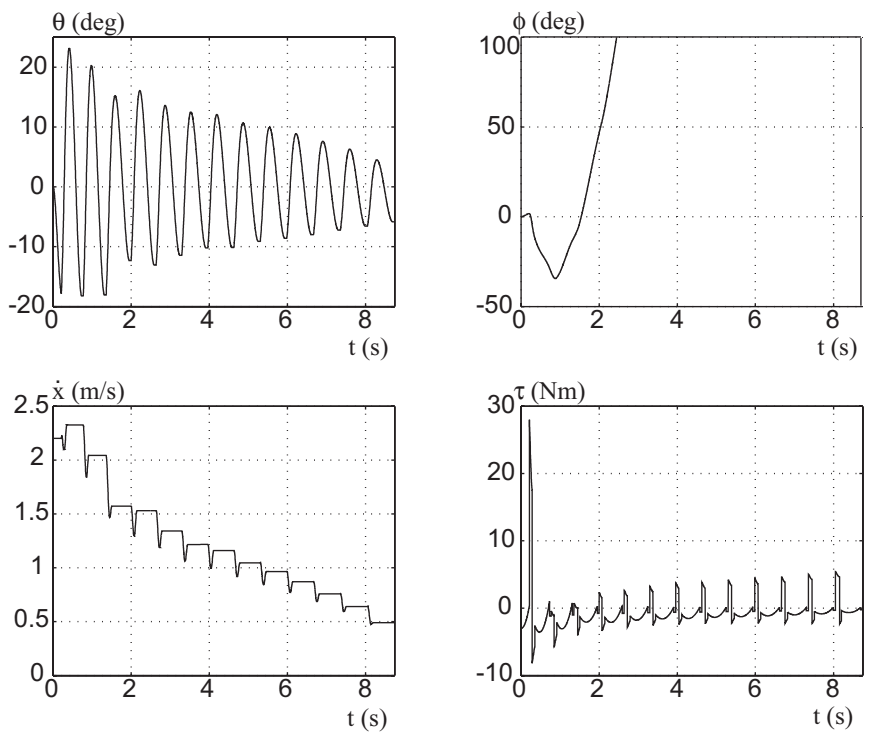

Fig. 5. Response of full model to SL-C.

Therefore, there is an indication that the SLIP model is not complete enough for dependable robot controller design and that future controllers should be designed using a more complete model. This conclusion is also verified by the form of the equations of motion, see Eq. (1). It is clear that, other than for the friction term, there is no coupling between the pitching motion and the leg angle or leg length. Therefore, a controller designed using the SLIP model cannot have a stabilizing effect on the pitching motion of a real robot. The rest of system destabilizes due to the leg inertia, not accounted for in SLIP based controllers. Because the leg inertia is not large, the system destabilizes more gradually.

Next, FL-C is applied to the full model, using the same robot parameters as in the previous case. It is desired to examine whether the modifications made to the initial controller now lead to a stable motion for the full model. The gains used in the full model controller are $k_{1}=0.2, k_{d}=5$, $k_{p}=10$. The response is shown in Fig. 6. As can be immediately seen, FL-C is successful in stabilizing the motion of the full model. There is a transient phase during which each cycle differs considerably from the previous one. This lasts for about $5 \mathrm{~s}$, after which the system enters a steady state motion.
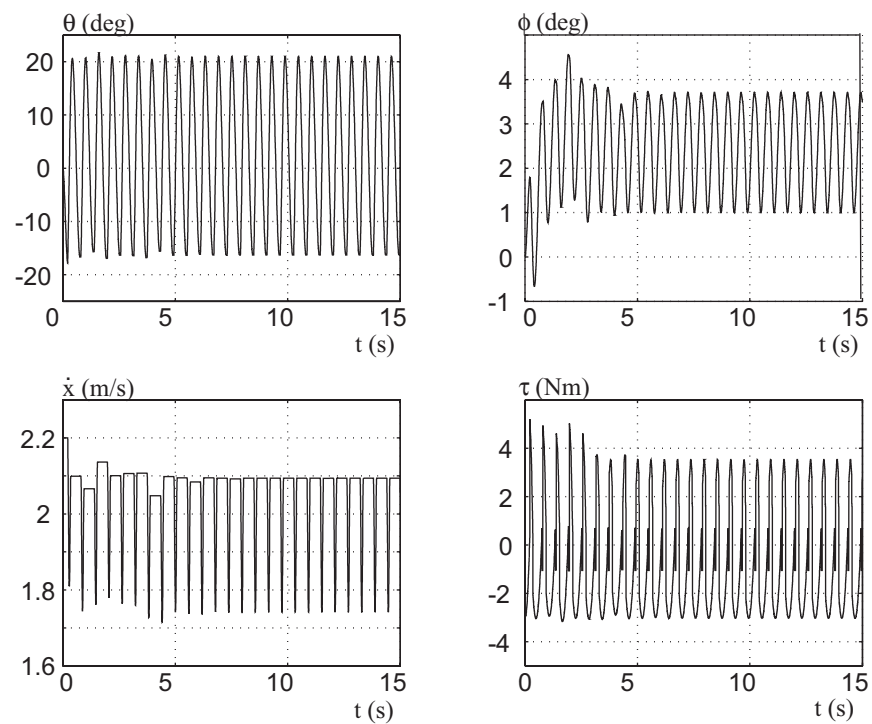

Fig. 6. Full robot model response to FL-C.

FL-C is now tested for various values of robot parameters, as well as for a range of initial conditions, to verify its generality. Note that all results presented below are for the same set of controller gain values used in the previous example.

This means that there is no need to adjust the controller, despite the changes in robot parameters and initial conditions used. As the stable time response always has the general form of Fig. 6, the test results are collected in tables.

Table 1 gives some sets of robot parameters and initial conditions $\dot{x}_{0}, y_{0}$ that lead to stable motions, as well as the resulting leg touchdown angle $\theta_{t d, s s}$ and maximum torque $\tau_{\text {max }, s s}$ of a random cycle, after the system has entered the steady state. The following robot parameters are kept the same: $m=10 \mathrm{~kg}, I_{b}=0.5 \mathrm{~kg} \mathrm{~m}^{2}, I_{l}=0.05 \mathrm{~kg} \mathrm{~m}^{2}, L=0.5 \mathrm{~m}, b_{h}=0.5$ $\mathrm{N} \cdot \mathrm{s} / \mathrm{m}$. The same typical initial velocity $\dot{x}_{0}=2 \mathrm{~m} / \mathrm{s}$ is used, so as not to vary too many parameters. 
Table 1. Robot parameters and initial conditions, and resulting stable gait characteristics.

\begin{tabular}{|c|c||c|c||c|c|}
\hline $\begin{array}{c}\mathrm{k} \\
(\mathrm{N} / \mathrm{m})\end{array}$ & $\begin{array}{c}b_{l} \\
(\mathrm{~N} \cdot \mathrm{s} / \mathrm{m})\end{array}$ & $\begin{array}{c}\dot{x}_{0} \\
(\mathrm{~m} / \mathrm{s})\end{array}$ & $\begin{array}{c}y_{0} \\
(\mathrm{~m})\end{array}$ & $\begin{array}{c}\theta_{t d, s s} \\
(\mathrm{deg})\end{array}$ & $\begin{array}{c}\tau_{\text {max,ss }} \\
(\mathrm{N} \cdot \mathrm{m})\end{array}$ \\
\hline \hline 5000 & 1 & 2.0 & 0.6 & -13.5 & 8.7 \\
\hline 5000 & 1 & 2.0 & 0.7 & -15.8 & 4.8 \\
\hline 9000 & 3 & 2.0 & 0.6 & -12.0 & 7.2 \\
\hline 9000 & 3 & 2.0 & 0.7 & -13.2 & 11.0 \\
\hline 12000 & 4 & 2.0 & 0.6 & -10.5 & 8.8 \\
\hline 12000 & 4 & 2.0 & 0.7 & -11.5 & 9.6 \\
\hline
\end{tabular}

It can be seen that FL-C results in steady motions for the full model, for a large range of the leg stiffness, as well as in the case of significant energy dissipation in the leg.

In Table 2, further examples of stable motions are given. In this table, various values of body inertia and mass are used to further demonstrate the controller's applicable range, still using the same gains as the first example of Fig. 6. Again, the initial conditions $\dot{x}_{0}, y_{0}$ are given, as well as the resulting leg touchdown angle $\theta_{t d, s s}$ and maximum torque $\tau_{\text {max,ss }}$ of a steady state cycle. The following robot parameters are kept steady: $k=9000 \mathrm{~N} / \mathrm{m}, I_{l}=0.05 \mathrm{~kg} \mathrm{~m}^{2}, L=0.5 \mathrm{~m}, b_{h}=0.5 \mathrm{~N} \cdot \mathrm{s} / \mathrm{m}$.

Table 2. Robot parameters and initial conditions, and resulting stable gait characteristics.

\begin{tabular}{|c|c|c||c|c||c|c|}
\hline $\begin{array}{c}\mathrm{m} \\
(\mathrm{kg})\end{array}$ & $\begin{array}{c}I_{b} \\
\left(\mathrm{~kg} \mathrm{~m}^{2}\right)\end{array}$ & $\begin{array}{c}b_{l} \\
(\mathrm{~N} \cdot \mathrm{s} / \mathrm{m})\end{array}$ & $\begin{array}{c}\dot{x}_{0} \\
(\mathrm{~m} / \mathrm{s})\end{array}$ & $\begin{array}{c}y_{0} \\
(\mathrm{~m})\end{array}$ & $\begin{array}{c}\theta_{t d, s s} \\
(\mathrm{deg})\end{array}$ & $\begin{array}{c}\tau_{\max , s s} \\
(\mathrm{Nm})\end{array}$ \\
\hline \hline 8 & 0.6 & 3 & 2.0 & 0.6 & -11.3 & 13.1 \\
\hline 8 & 0.6 & 3 & 2.0 & 0.7 & -13.0 & 4.3 \\
\hline 15 & 0.8 & 2 & 2.0 & 0.6 & -14.4 & 3.9 \\
\hline 15 & 0.8 & 2 & 2.0 & 0.7 & -16.5 & 11.0 \\
\hline 20 & 1.5 & 1 & 2.0 & 0.6 & -15.5 & 3.8 \\
\hline 20 & 1.5 & 1 & 2.0 & 0.7 & -17.0 & 6.2 \\
\hline
\end{tabular}

Accomplishing steady motions using such a wide range of parameters and the same controller gains is made possible by the systematic exploration of the energy transfer mechanism. Because of this, the desired leg angle at touchdown is an analytical expression of the robot parameters, as seen in Section IV. As a result, once a set of reasonable gains is found for the controller, it is extremely accommodating to changes in both initial conditions and robot parameters. This makes the controller novel, as previous attempts to use the leg touchdown angle as a control input on realistic robots have relied on trial and error [11]. Also a number of previous controllers, such as [6], used special initial conditions so that the robot was already close to the steady state cycle at the beginning of its motion. It is evident that our controller, requiring only knowledge of the initial conditions, is capable of overcoming significant transient effects and still leading to stable locomotion. Finally, the controller can be implemented using only one actuator, situated at the hip, providing a real advantage in construction over the two-actuator implementation.

\section{CONCLUSIONS}

In this paper the mechanism of energy transfer was studied, between the single actuated DOF of a one-legged hopping robot and the remaining unactuated DOFs, during stable locomotion. The concept of the energy transfer mechanism was analyzed, followed by an analytical study of the mechanism. Using this analysis, an initial controller for the simple SLIP model was found. The model incorporated friction in the leg and hip, and used a single actuator at the hip. Although this controller was successful for the SLIP model, it did not lead to stability for the full robot model. This result provides a strong indication that the SLIP model, often used for controller design, may be unsuitable for this purpose. Modifications were then made to the initial controller that resulted in stabilizing the full model for a wide range of parameters, using only a single actuator.

\section{REFERENCES}

[1] W. J. Schwind and D. E. Koditschek, "Approximating the Stance Map of a 2-DOF Monoped Runner", Journal of Nonlinear Science, vol.10, pp. 533-568, 2000.

[2] W. J. Schwind and D. E. Koditschek, "Characterization of Monoped Equilibrium Gaits", Proc. 1992 IEEE Int. Conference on Robotics and Automation, pp. 1986-1992, 1997.

[3] I. Poulakakis, E. Papadopoulos and M. Buehler, "On the Stable Passive Dynamics of Quadrupedal Running", Proc. 2003 IEEE Int. Conference on Robotics \& Automation, Taipei, Taiwan, pp.1368-1373, Sept. 2003.

[4] R. Dummer and M. Berkemeier, "Low-Energy Control of a One-Legged Robot with 2 Degrees of Freedom", Proc. 2000 IEEE Int. Conference on Robotics \& Automation, San Francisco, CA, pp. 2815-2821, April 2000.

[5] W. J. Schwind and D. E. Koditschek, "Control of Forward Velocity for a Simplified Planar Hopping Robot”, Proc. 1995 IEEE Int. Conference on Robotics \& Automation, Nagoya, Aichi, Japan, pp.691-696, 1995.

[6] M. Ahmadi and M. Buehler, "Stable control of a simulated one-legged running robot with hip and leg compliance", IEEE Transactions on Robotics and Automation, vol. 13, issue 1, pp. 96 -104, 1997.

[7] C. Francois and C. Samson, "Running with Constant Energy", Proc. 1994 IEEE International Conference on Robotics and Automation, pp. 131-136, 1994.

[8] M. H. Raibert, "Legged Robots That Balance”, MIT Press, Cambridge, MA, 1986.

[9] M. Ahmadi and M. Buehler, "The ARL monopod II running robot: control and energetics", Proc. 1999 IEEE International Conference on Robotics and Automation, vol. 3, pp. 1689 -1694, 1999.

[10] P. Gregorio, M. Ahmadi and M. Buehler, "Design, Control, and Energetics of an Electrically Actuated Legged Robot", IEEE Transactions on Systems, Man and Cybernetics, 27B(4), pp. 626-634, 1997.

[11] S. Talebi, I. Poulakakis, E. Papadopoulos and M. Buehler, "Quadruped Robot Running With a Bounding Gait", Proc. $7^{\text {th }}$ Int. Symp. on Experimental Robotic (ISER'00), Honolulu, HI, pp.281-289, December 2000

[12] D. Papadopoulos and M. Buehler, "Stable Running in a Quadruped Robot with Compliant Legs", Proc. 2000 IEEE Int. Conf. Robotics and Automation, San Francisco, CA, pp. 444-449, April 2000.

[13] E. Papadopoulos and N. Cherouvim, "On Increasing Energy Autonomy for a One-Legged Hopping Robot", Proc. 2004 IEEE Int. Conf. on Robotics and Automation, New Orleans, LA, pp. 4645-4650, April 2004. 